\title{
Phytoplankton population along certain Egyptian coastal regions of the Red Sea
}

\author{
Fedekar F. Madkour ${ }^{1}$; Mohsen M. El-Sherbiny ${ }^{2}$ and Maher A. Aamer ${ }^{2}$ \\ 1- Marine Science Department, Faculty of Science, Port Said University, Egypt \\ 2- Marine Science Department, Faculty of Science, Suez Canal University, \\ Egypt
}

\begin{abstract}
Curface phytoplankton samples were collected at eight sites along certain Egyptian coasts of the Red Sea located at the southern of Sinai Peninsula and the two gulfs (Gulf of Suez and Gulf of Aqaba). Qualitative and quantitative estimation of phytoplankton species were conducted seasonally during the period from autumn 2007 to summer 2008. The phytoplankton population was fairly diversified (181 species) comprised mainly two groups; dinoflagellates (116 species) and diatoms (60 species). The dominant genera, in terms of the number of species, were Ceratium, Protoperidinium, Dinophysis and Gonyaulax for dinoflagellates, while Chaetoceros, Rhizosolenia and Nitzschia for diatoms. The phytoplankton standing crop was relatively low with and average seasonal density of $1.4-7.7 \times 10^{3}$ individual/l, reflecting the oligotrophic conditions of this area. The phytoplankton abundance showed one peak in summer when Hemiaulus hauckii $\left(9 \times 10^{3}\right.$ individual/l) from diatoms and Trichodesmium spp. $\left(3 \times 10^{3}\right.$ individual/l) from blue-green algae comprised the main bulk of the phytoplankton standing crop. Relatively high production of different species appeared at different sites during specific season, such as Nitzschia closterium, Pseudo-nitzschia delicatissima, Rhizosolenia alata and $R$. calcar-avis. On the other hand, there were relatively low variations in phytoplankton composition the along study area. Spatial distribution of phytoplankton showed that Gulf of Suez differes in the dominant species and timing of abundance from both Gulf of Aqaba and the southern sites of Sinai Peninsula.
\end{abstract}

Key words: Phytoplankton, species composition, Gulf of Suez, Gulf of Aqaba, Red Sea.

\section{INTRODUCTION}

The Red Sea is a long narrow basin which extends over $1900 \mathrm{~km}$ north of the narrow straits of Bab-el-Mandab between $30^{\circ} \mathrm{N}$ to $12^{\circ} 30^{\prime} \mathrm{N}$. The Sinai Peninsula divides the northern extremity into the shallow Gulf of Suez and the deep Gulf of Aqaba. There is almost no rainfall as any permanent rivers inflow into the Red Sea, and evaporation is high (Edwards, 1987). Salinity values increase from south to north with decreasing surface temperatures (Edwards, 1987). In the northern Red Sea, the average surface temperature fluctuates between $21.3^{\circ} \mathrm{C}$ and $27.9^{\circ} \mathrm{C}$ showing slightly greater amplitude in the Gulf of Suez $\left(17.9^{\circ} \mathrm{C}\right.$ to $26.5^{\circ} \mathrm{C}$ ) (Morcos, 1970$)$. Surface salinity reaches up to $40-41 \%$ 
in the northern Red Sea and the Gulf of Aqaba, and to more than 41\%o in the Gulf of Suez (Halim, 1969). The northern Red Sea is permanently stratified throughout the year, mixing depth is $<100 \mathrm{~m}$, a deep chlorophyll maximum is present year-round at about $80-100 \mathrm{~m}$ depth, and the euphotic depth is well beyond $100 \mathrm{~m}$ (Pätzold et al., 2000).

Previous studies on the phytoplankton population of the Red Sea introduced some information about the phytoplankton species composition and community structure. In oceanic water of the central Red Sea, Halim (1969) reported 209 species (125 dinoflagellates and 84 diatoms) and Skaikh et al. (1986) recorded 283 species (of them, 110 dinoflagellates and 137 diatoms). At Saudi Arabia coasts, Dowidar et al., (1978) recorded about 224 species in the region of Obhor, Jeddah (111 dinoflagellates and 112 diatoms). Recently, Touliabah et al. (2010) studied phytoplankton composition at Jeddah coast in relation to some ecological factors and recorded 73 species. Few studies have been carried out on the Gulf of Aqaba, some concerning seasonal dynamics of phytoplankton (Al-Najjar et al., 2007) or the primary production (Sournia, 1977; Levanon-Spanier et al., 1979). In the Gulf of Suez, the phytoplankton composition and distribution in relation to environmental factors was studied by Deyab et al. (2004) and Ismail (2005).

Most of the previous studies reported that late winter-early spring is characterized by high phytoplankton production throughout the Red Sea, resulting from the winter monsoon (Halim, 1969; Levanon-Spanier et al., 1979). Other studies observed bimodal pattern with a higher one in December-February and a smaller one in June-August (Skaikh et al., 1986). The flourishing of Trichodesmium spp. is a known phenomenon along the Red Sea during summer as reported in Saudi coasts (Dowidar et al., 1978) and in the Gulf of Aqaba (Kimor and Golandsky, 1977; Gordon et al., 1994; Post et al., 2002). Occurrence of some diatoms such as Hemiaulus and Rhizosolenia in adequate high numbers associating with Trichodesmium was reported from the Gulf of Aqaba (Post et al., 2002). These species often associate cyanobacterial symbionts capable of nitrogen fixation (Gordon et al., 1994). In the Gulf of Aqaba, phytoplankton is dominated by ultra- and picoplankton (Sommer, 2000; Sommer et al., 2002).

Studies on the phytoplankton population of the Red Sea still remain inadequate when compared to the size and ecological importance of this area. The present paper aims to study the seasonal changes in phytoplankton composition and standing crop distribution in the neritic waters of the Egyptian Red Sea coasts, including the Gulf of Suez and the Gulf of Aqaba.

\section{MATERIALS AND METHODS}

In the present study, surface water samples were collected seasonally between autumn 2007 and summer 2008 at eight sites along the Egyptian Red Sea coasts (Fig. 1). Sites 1 and 2 represent the Gulf of Suez locating in front of 
El-Tor and at the entrance of the gulf, respectively. Sites 3-6 are situated around the southern tip of Sinai Peninsula. Site 3 lies in front of Ras Mohamed; sites 4 and 5 are situated at a semi-enclosed coastal bay (Sharm El-Maya) and site 6 is located off Sharm El-Sheikh city. Sites 7 and 8 represent the Gulf of Aqaba, the former located at southern entrance of the gulf and the later lies in front of Dahab. At each site, water temperature and dissolved oxygen were measured in situ by an ordinary thermometer and oxygen meter (JENWAY, Model 9070), respectively. Nutrient salts (nitrate, nitrite, phosphate and silicate) were measured spectrophotometrically following the methods of Parsons et al. (1984). For chlorophyll $a$ estimation, 5 liters of seawater were filtered using $0.45 \mu \mathrm{m}$ membrane filters, extracted in $90 \%$ acetone and measured spectrophotometrically according to Parsons et al. (1984).

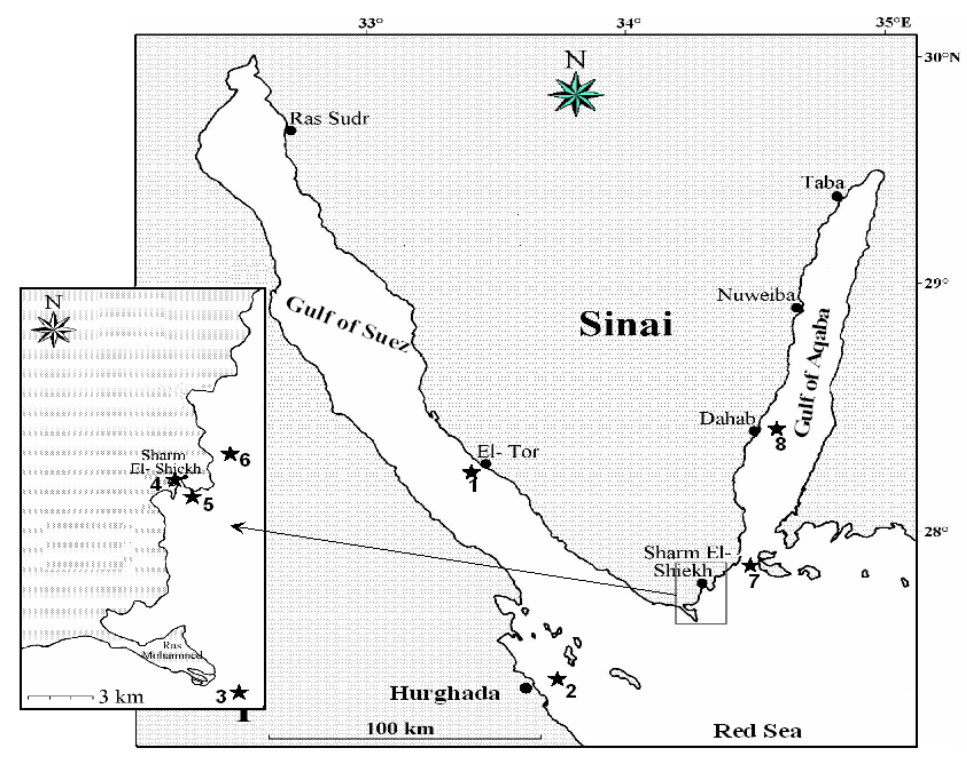

Fig. 1: Sampling sites along certain Egyptian coasts of the Red Sea.

For phytoplankton investigation, 10 liters of surface water were collected, preserved in $4 \%$ neutral formalin and concentrated by decanting. Sub-sample of $10 \mathrm{ml}$ was settled in a counting chamber of Hydrobios inverted microscope and cell count was performed using Utermöhl's technique (Utermöhl, 1958). The entire chamber bottom was scanned at $10 \mathrm{X}$ for large and/or rare species. The smaller and dominant species were counted at magnifications of $20 \mathrm{X}$ and counts were expressed as individual/l. Many keys and reference books were used for identification (Hendey, 1964; Taylor, 1976; Dodge, 1982; Tomas, 1996). Similarity matrix (S) was performed to deduce the variation between sites. The 
similarity coefficient was calculated by the Bray and Curtis formula (Field et al., 1982) using PRIMER program Version 5.2.

\section{RESULTS}

The values of measured physico-chemical parameters and chlorophyll $a$ at the studied sites were averaged for the three regions as follows: The Gulf of Suez (sites 1 and 2), the southern of Sinai Peninsula (sites 3-6) and the Gulf of Aqaba (sites 7 and 8), and are shown in Table (1).

Table 1: The average values of studied physico-chemical parameters and chlorophyll $a$ at Gulf of Suez (I), southern of Sinai Peninsula (II), and Gulf of Aqaba (III) sites during autumn 2007- summer 2008.

\begin{tabular}{|c|c|c|c|c|c|c|c|c|c|c|c|c|}
\hline Seasons & \multicolumn{3}{|c|}{ Autumn } & \multicolumn{3}{|c|}{ Winter } & \multicolumn{3}{|c|}{ Spring } & \multicolumn{3}{|c|}{ Summer } \\
\hline Sites & I & II & III & I & II & III & I & II & III & $\mathbf{I}$ & II & III \\
\hline Temperature $\left({ }^{\circ} \mathrm{C}\right)$ & 26.1 & 26.1 & 25.5 & 22.1 & 21.8 & 21.6 & 22.8 & 23.6 & 23.5 & 29.8 & 30.9 & 29.6 \\
\hline DO (mg/l) & 6.9 & 7.2 & 7.3 & 7.2 & 7.3 & 7.4 & 7.0 & 7.1 & 6.9 & 6.4 & 6.6 & 6.5 \\
\hline Nitrate $(\mu \mathrm{M})$ & 0.42 & 0.48 & 0.38 & 0.09 & 0.11 & 0.07 & 1.25 & 1.40 & 0.91 & 0.94 & 1.13 & 0.91 \\
\hline Nitrite $(\mu \mathrm{M})$ & 0.011 & 0.013 & 0.010 & 0.012 & 0.012 & 0.013 & 0.110 & 0.113 & 0.095 & 0.025 & 0.035 & 0.020 \\
\hline Phosphate $(\mu \mathrm{M})$ & 0.015 & 0.015 & 0.013 & 0.300 & 0.328 & 0.210 & 0.610 & 0.718 & 0.500 & 0.205 & 0.443 & 0.240 \\
\hline Silicate $(\boldsymbol{\mu M})$ & 1.20 & 1.75 & 1.08 & 2.00 & 2.96 & 1.55 & 0.38 & 0.61 & 0.38 & 0.18 & 0.25 & 0.15 \\
\hline$(\mu \mathrm{g} / \mathrm{l})$ & 0.115 & 0.145 & 0.125 & 0.125 & 0.150 & 0.120 & 0.155 & 0.148 & 0.125 & 0.960 & 0.963 & 0.900 \\
\hline
\end{tabular}

In the investigated area, temperature and dissolved oxygen showed low variation among sites. Surface water temperature ranged between the minimum average value of $21.6{ }^{\circ} \mathrm{C}$ in winter and the maximum of $30.9{ }^{\circ} \mathrm{C}$ in summer, while dissolved oxygen fluctuated between $6.4 \mathrm{mg} / \mathrm{l}$ at the Gulf of Suez in summer and $7.4 \mathrm{mg} / \mathrm{l}$ at the southern of Sinai Peninsula in winter. Low nutrient concentrations were observed throughout the whole year with the highest values at the southern of Sinai Peninsula. The averages of nutrients were fluctuated between minima of $0.07,0.010,0.013$ and 0.15 , and maxima of 1.40, 0.113, 0.718 and $2.96 \mu \mathrm{M}$ for nitrate, nitrite, phosphate and silicate, respectively. Chlorophyll $a$ was rather low throughout the whole study area. Seasonally, summer was the most productive season giving a maximum chlorophyll a value of $0.963 \mu \mathrm{g} / \mathrm{l}$ at the southern of Sinai Peninsula, while low values was detected in the other seasons, particularly in autumn.

The phytoplankton species recorded in the present study was 181 species comprised mainly of dinoflagellates and diatoms (Table 2). Among dinoflagellates, 116 species belonging to 21 genera were recorded. Of them, Ceratium was the most important genus in terms of the number of species (34 species, 29\% of the dinoflagellates) followed by Protoperidinium (21 species, $18 \%$ of the dinoflagellates). Diatoms were represented by 60 species belonging to 28 genera. The most diversified genera were Chaetoceros (9 species) and Rhizosolenia (8 species). In addition, 2 species of Cyanobacteria, 2 species 
(from 2 genera) of coccolithophores and 1 species of silicoflagellates were recorded. The diversity of phytoplankton varied among sites and seasons. The highest species number was recorded at site 3 with a total number of 130 species (85 dinoflagellates and 41 diatoms), followed by sites 7 and 8 (Gulf of Aqaba). The lowest number of phytoplankton species was recorded at site 1 (Gulf of Suez) with a total number of 88 species (46 dinoflagellates and 40 diatoms).

As shown in Figure (2), the phytoplankton population was more diversified in autumn particularly at sites 3 and 7 (106 and 105 species, respectively) followed by summer especially at site 7 (94 species). Dinoflagellates displayed high diversity all over the year except in winter recording the highest number of species in summer at site 7 (75 species). On the other hand, the highest number of diatoms appeared in winter while low numbers were recorded in spring and summer.

Table 2: Phytoplankton species recorded at certain Egyptian coasts of the Red Sea during autumn 2007-summer 2008.

\begin{tabular}{|c|c|c|c|c|c|c|c|c|c|c|c|c|c|c|c|c|c|}
\hline Fhytoplankton Species/Sites & 1 & 2 & 3 & 4 & 5 & 6 & 7 & 8 & Plytoplankton Species/Sites & 1 & 2 & 3 & 4 & 5 & 6 & 7 & 8 \\
\hline Dingphyceae & & & & & & & & & C. triesos (0. F. Muller) Natsch & * & * & * & $*$ & * & H & H* & H \\
\hline $\begin{array}{l}\text { Ampinsolevio bidentato Schroder } \\
\text { Ceration arietinum Cleve } \\
\text { C belone Cleve }\end{array}$ & H & H & $\begin{array}{l}\text { H } \\
4 \\
k\end{array}$ & & $\begin{array}{l}* \\
* \\
*\end{array}$ & k & * & $\begin{array}{l}\text { k } \\
k \\
k\end{array}$ & $\begin{array}{l}\text { C. vuitur Cleve } \\
\text { Ceratocory anmato (Schult) Kof oid } \\
\text { C. goureti Paulsen }\end{array}$ & * & $\begin{array}{l}\text { * } \\
\text { * }\end{array}$ & $\begin{array}{l}\text { k } \\
4 \\
k\end{array}$ & & & H & $\begin{array}{l}* \\
* \\
*\end{array}$ & \\
\hline C breve (0stenfeld \& Schm) Schroder & * & $+k$ & & & * & k & * & k & C. harida Stein & * & & * & * & H & & H & k \\
\hline 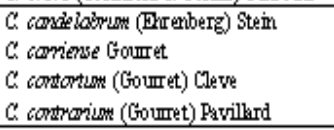 & $\begin{array}{l}\text { * } \\
k \\
*\end{array}$ & $\begin{array}{l}k \\
* \\
* \\
*\end{array}$ & $\begin{array}{l}* \\
k \\
k \\
*\end{array}$ & $\begin{array}{l}* \\
k \\
k \\
*\end{array}$ & *: & $\begin{array}{l}* \\
*\end{array}$ & $\begin{array}{l} \\
4 \\
4 \\
4\end{array}$ & * & $\begin{array}{l}\text { Cuharistes regus Ste in } \\
\text { Dinopiysis argus Ste in } \\
\text { D. cauriata Saville \& Kert } \\
\text { D. cureus Schull }\end{array}$ & * & * & H & $*$ & * & H* & * & * \\
\hline C dacbinatum Karst. & & * & $*$ & * & "k & * & $*$ & * & D. doryphorum 9en & & "k & H & & k & H & * & * \\
\hline 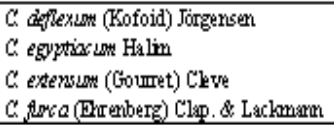 & $\begin{array}{l}\text { k } \\
*\end{array}$ & $\begin{array}{l}* \\
*\end{array}$ & $\begin{array}{l}* \\
*\end{array}$ & * & $*$ & $\begin{array}{l}k \\
k\end{array}$ & * & * & $\begin{array}{l}\text { D. wiles Chve } \\
\text { D. operculata Ste in } \\
\text { D. parvila Schutt } \\
\text { D. porodicturn Stein }\end{array}$ & & & * & H & & H & * & \\
\hline C. fusus (EFrenberg) Duj. & * & * & * & * & * & * & * & * & D. raga Stein & & & & & * & & & \\
\hline $\begin{array}{l}\text { C gravidow Goumet } \\
\text { C haridum Gran } \\
\text { C humile Jörgensen }\end{array}$ & $\begin{array}{l}\text { * } \\
*\end{array}$ & $\begin{array}{l}* \\
k \\
k\end{array}$ & $\begin{array}{l}\text { H } \\
\text { H }\end{array}$ & & $\begin{array}{l}* \\
*\end{array}$ & $\begin{array}{l}* \\
*\end{array}$ & $\begin{array}{l}* \\
*\end{array}$ & $\begin{array}{l}\text { H } \\
\text { H* }\end{array}$ & $\begin{array}{l}\text { D. sphaerica Ste in } \\
\text { D. tripos Gourlet. } \\
\text { D. upacatha Se in }\end{array}$ & $\begin{array}{l}\text { H } \\
\text { H. }\end{array}$ & H. & & $*$ & * & $\begin{array}{l}k \\
k \\
k\end{array}$ & & \\
\hline C ṗxisun (Karsten) Jörgensen & & * & & k & * & H & * & H* & Goriodowapodyeciricun (Pouch.) Jörgensen & & H. & H & H & k & H* & H & \\
\hline C miflatum (Kofoid \& borgensen & & & H & & & H & & k & G. sphaericum Mirray \& whitting & & H & * & * & H & k & * & H \\
\hline $\begin{array}{l}\text { C korstemï Paviikrd } \\
\text { C kojoidi Jörgensen } \\
\text { C breatin (Errenberg) Clewe }\end{array}$ & $\begin{array}{l}* \\
*\end{array}$ & * & $\begin{array}{l}* \\
*\end{array}$ & * & * & $\begin{array}{l}\text { k } \\
k \\
*\end{array}$ & * & $*$ & $\begin{array}{l}\text { Goryaulax digensis Kofoid } \\
\text { G. iyalina 0sterfeld \& Schmidt } \\
\text { G. kofoidi Prvillard }\end{array}$ & * & $\begin{array}{l}* \\
*\end{array}$ & * & $*$ & $*$ & $*$ & $\begin{array}{l}* \\
4 \\
*\end{array}$ & $\begin{array}{l}* \\
* \\
*\end{array}$ \\
\hline C. maroceros (Ekrenberg) Varh. & & H & 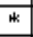 & H & 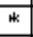 & * & * & * & G. winuto Kof oid \& Michener & & & * & $*$ & & & * & * \\
\hline $\begin{array}{l}\text { C massilizse (Gounet) Jörgensen } \\
\text { C minutım Jorgensen } \\
\text { C panillad Jörgensen }\end{array}$ & $\begin{array}{l}* \\
*\end{array}$ & $\begin{array}{l}\text { H } \\
\text { H }\end{array}$ & $\begin{array}{l}\text { H } \\
\text { H }\end{array}$ & & * & $\begin{array}{l}* \\
k \\
k\end{array}$ & * & $\begin{array}{l}\text { H } \\
*\end{array}$ & $\begin{array}{l}\text { G. ovais Schiller } \\
\text { G. poby growwa Stein } \\
\text { G. scrisso Kofoid }\end{array}$ & & & $\begin{array}{l}* \\
k \\
k\end{array}$ & $\begin{array}{l}k \\
*\end{array}$ & & * & $\begin{array}{l}\text { H. } \\
\text { H. }\end{array}$ & $\begin{array}{l}* \\
* \\
*\end{array}$ \\
\hline C pentagoromon foumet. & * & & * & & * & & & & G. sprinfera (Clap $\&$ Lachmann) Diesing & & & $*$ & * & $*$ & & * & \\
\hline C platycone Daday & & & & & k & H & & & G. tumbynt MIIry \& Whitting & * & & H & & H & & * & \\
\hline $\begin{array}{l}\text { C pulcheñun Jörgensen } \\
\text { C reflexum Cleve } \\
\text { C sionidì Jörgensen }\end{array}$ & $\begin{array}{l}\text { * } \\
*\end{array}$ & $\begin{array}{l}\text { k } \\
*\end{array}$ & * & & * & & $*$ & * & 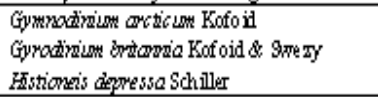 & * & & $\begin{array}{l}* \\
k \\
k\end{array}$ & & $\begin{array}{l}* \\
*\end{array}$ & $\begin{array}{l}* \\
*\end{array}$ & $\begin{array}{l}\text { H } \\
*\end{array}$ & $\begin{array}{l}* \\
*\end{array}$ \\
\hline C teres Kofoid & * & * & * & H & * & * & * & * & H longicolds Kofoid & & & & & & & & H: \\
\hline $\begin{array}{l}\text { C trichoxeros (Evrerberg) Kof oid } \\
\text { O. constrictum (Ste in) Butschli } \\
\text { 0. frenguelui Rampi }\end{array}$ & * & H & $\begin{array}{l}k \\
4\end{array}$ & & * & $\begin{array}{l}k \\
*\end{array}$ & * & H & $\begin{array}{l}\text { Oxytoxun areolatum Rmpi } \\
\text { P. sphaenicum (MLn.\& Whitt.) Balech } \\
\text { P. steinii Jörgensen }\end{array}$ & * & H. & * & & * & $\begin{array}{l}* \\
k\end{array}$ & $\begin{array}{l}* \\
k \\
k\end{array}$ & $\begin{array}{l}\text { H. } \\
\text { H. }\end{array}$ \\
\hline 0. gianiolus Stein & & & & & & & * & k & Pyrogstis fustorow is Thompson \& Munay & * & H. & H & * & H & H & * & H \\
\hline
\end{tabular}


Table 2: Continued.

\begin{tabular}{|c|c|c|c|c|c|c|c|c|c|c|c|c|c|c|c|c|c|}
\hline O. constrictum (Stein) Butschli & & & t & * & & * & & & P. sphaeticum (Murr.\& Whitt) Balech & & * & & & & * & * & * \\
\hline O. frenguellii Rampi & & * & & & & & & * & P. steinii Jörgensen & * & & * & & * & + & * & \# \\
\hline O. gladalus Stein & & & & & & & t & \# & Ayrocystis fusifomis Thompson \& Murray & * & * & * & * & * & \# & * & * \\
\hline O. milneri Murray \& Whitting & & & * & & & & * & * & P. hamulus Cleve & & * & * & & * & * & * & * \\
\hline O. scolopax Stein & & * & * & * & * & * & * & * & P. lunule (Schutt) Schutt & & & * & * & * & * & * & * \\
\hline O. sphaeroideum Stin & & & & & & & & * & P. pseudonoctiluca Thompson Murray & & * & * & * & * & * & * & * \\
\hline O. tesselatum Schutt & & & * & * & * & & & & Ayrodinium schilleni (Matz.) Schiller & * & & * & * & * & * & * & \\
\hline Palaeophalactoma unicinctum Schiller & * & * & * & * & * & * & * & * & Pyrophacus hotologium Stein & & & * & * & & & * & t \\
\hline Peridiniopsis asymmetrica Mangin & * & & * & * & * & * & & * & P. steinn (Schiller) Whallet Dale & & & * & * & & & & \\
\hline P. lenticula Stein & * & & & & & & & & Scrippsiela faetonies (Pauls.) Balech \& Soares & & & * & * & * & * & * & * \\
\hline Podolampas bipes Stein & & * & * & & * & * & & & S. trochoidea (Stein) Loeb. III & & * & * & * & * & * & * & * \\
\hline P. palmipes Stein & & * & * & * & * & & * & * & Diatoms & & & & & & & & \\
\hline Prorocentnum compressum (Bail) Abe exDodge & * & * & * & * & * & * & * & * & Actinopychus undulatus (Bail) Ralfs & & * & * & & & & & \\
\hline P. gracile Schutt & * & * & * & * & * & * & * & * & Amphora coefaeformis Agardh & & & * & & & & & \\
\hline P. maximum Schiller & * & + & * & * & * & * & * & * & Campylodiscus déconus Breb. & + & & & + & & & & \\
\hline P. micans Ehrenberg & & * & * & * & * & * & * & * & Chaetoceros compressum Lauder & * & * & * & * & * & * & * & * \\
\hline P. obtusa Pavilland & * & & * & * & * & * & * & * & C brevis Schutt & * & * & * & * & \# & * & * & * \\
\hline Protoceratium aereolatum Kofoid & & & & * & & & & & C coarctatus Lauder & * & * & & * & * & & * & \# \\
\hline P. reticulatum Buetschli & & * & & & * & * & * & & C cutvisetum Cleve & * & * & * & & * & * & * & * \\
\hline Protoperidinium brochii Kofoid \& Swery & & * & * & * & * & * & * & & $C$ decipiens Cleve & * & * & * & * & * & * & * & * \\
\hline P. cerasus Paulsen & & * & + & * & + & * & & * & C laciniosus Schütt & * & * & * & * & * & * & * & * \\
\hline P. conicum 0stenfeld \& Schm & & & * & & * & & * & * & C lauderi Ralfs & * & * & * & * & * & * & * & * \\
\hline P. crassipes Kofoid & * & * & * & * & * & * & * & * & C lorenzianum Grunow & * & * & * & * & * & * & t & * \\
\hline P. cutvipes Ostenfeld & & * & * & & & & * & * & C tetrastichon Cleve & t & * & * & t & \# & 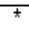 & t & * \\
\hline P. depressum Bailey & & * & * & * & * & * & * & * & Climacodum fraunfeldanum Grunow & & & * & & * & & & * \\
\hline P. diabolus Cleve & & * & * & * & * & * & * & * & Cocconeis scutellum Ehrenberg & t & & * & * & & & & \\
\hline P. divergens Ehrenberg & * & * & * & * & + & * & * & * & Coreth ron cyyophylum Castracane & & & & & & * & & \\
\hline P. excentricum Paulsen & * & * & * & * & * & & * & * & Cosciodiscus centralis Ehrenherg & * & * & * & * & \# & * & * & * \\
\hline P. globulus Stein & & * & * & * & * & + & * & * & Ceccentricus Ehrenberg & * & & & * & + & \# & + & \\
\hline P. grande Kofoid & & & & & * & & & * & C lineatus Ehrenberg & & * & * & & & * & & \\
\hline P. granii (Ostenfeld) Paulsen & & & * & & * & & & * & C oculus-indss Ehrenherg & * & * & * & & & * & * & * \\
\hline P. leonis Pavillard P. $\mathbf{n}$ & * & & * & * & * & * & * & * & C perforatus Ehrenberg & & t+ & * & & & + & * & \\
\hline P. oceanicum Vanhoffen & * & + & * & * & * & * & & * & C. radiatus Ehrenberg & & * & * & * & * & * & * & * \\
\hline P. ovatum Pouchet & & & * & & * & & * & * & Diplonies crabro (Ehrenberg) Wm. Smith & & * & * & * & * & * & * & * \\
\hline
\end{tabular}

Table 2: Continued.

\begin{tabular}{|c|c|c|c|c|c|c|c|c|c|c|c|c|c|c|c|c|c|}
\hline P. ovatum Pouchet & & & * & & t & & * & * & Diplonies crab to (Ehrenherg) Wm. Smith & & t & * & t & t & t & * & * \\
\hline P. palidum 0stenfld & & & * & * & * & t & t & + & Guinatdia flaccida (Cast). H. Peragallo & * & & & & & & & \\
\hline P. pedunculatum Schutt & & * & * & * & t & * & & & Grosigma balticum (Ehrenberg) Cleve & * & & & & * & * & & \\
\hline P. pelludidum Bergh & & * & * & * & * & * & & 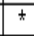 & Hemiaulushauchii Grunow & * & + & * & * & * & * & * & * \\
\hline P. pentagonum (Gran) Balech & & & & * & & & & & H. membranaceous Cleve & & * & & & & & & \\
\hline H. senensis Greville & & & & & & * & t & t & R stolterfothii H Peragallo & & * & & & & t & + & * \\
\hline Leptopylindus danicus Cleve & * & * & * & * & * & * & * & * & R sylifomis Brightwell & * & * & + & + & * & * & & * \\
\hline Navicula membranacea Cleve & & & * & * & + & * & 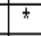 & + & Schroderella delicatula (H. Prgallo) Pavillard & * & & & t & & * & t & * \\
\hline Mitzschia closterium (Ehrenherg) W. Snith & t & * & * & t & t & * & * & t & Striatella univanctata (Lyngbye) Agardh & t & & & & & & & * \\
\hline N. hungatica Grunow & * & * & * & * & * & & & * & Surrivella fastuosa Ehrenberg & & t & t & & & & & \\
\hline N. longisima (Breb.) Ralfs & * & * & & * & & t & & & S. gemma Ehrenherg & & & & & * & & & t \\
\hline N. lorenziana Grunow & * & * & * & * & t & & 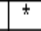 & * & S. ovalis Breb. & t & & & & & & * & \\
\hline N. pandurformis Gregory & * & & 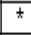 & & & & & * & Simedra ulna (Nizsch) Ehrenherg & * & * & & * & * & & * & \\
\hline Pinnulana trevelyana Donlin & * & & t & & & & & t & S. crystalina (Agardh) Kutz. & t & t & * & t & t & t & * & \\
\hline Planktoniella sol (Wallich) Schutt & & * & * & t & * & * & * & + & Thalasionema nitzschiades Grunow & t & & t & t & t & & * & \\
\hline Pleurosigma angulatum Quelett & & * & * & t & & * & * & t & Thalasiositra decipiens (Grunow) Jörgensen & & & & & & * & t & \\
\hline P. elongatum W. Smith & t & & * & & & & t & & Thalasiothix longasima Cleve \& Grunow & t & & t & & & & & \\
\hline P. nomanii Ralfs & & t & t & & t & & t & * & T. frauenfeldii (Grunow) Cleve\&Grunow & & t & * & * & & * & & \\
\hline Pseudo-nitzschia delicatisima (Celve) Heiden & * & & * & * & & & * & + & Cyanobacteria & & & & & & & & \\
\hline Rhabdonema adraticum Kutzing & & * & & & & & t & & Trichodesmium eyth raeum Ehrenherg \& Gomont & * & * & * & * & * & * & * & \\
\hline Rhizosolenia alata Brightwell & * & t & * & * & * & * & + & * & Trichodesmium sp. & * & + & t & 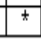 & * & + & & * \\
\hline R.calcar-avis M. Schultze & * & * & * & & * & * & t & * & Coccolithophores & & & & & & & & \\
\hline R hebetata Bailey & t & * & t & t & t & * & & * & Syracosphaera mediterranea Lohmann & & & & & t & * & & \\
\hline R imbricata Breightwell & t & & & & & & & t & Coronosphaera sp. & & & * & * & & & & * \\
\hline R indica H. Peragallo & * & * & & & & * & * & & Silicoflagellates & & & & & & & & \\
\hline R setigera Brightwell & t & * & * & * & * & & & t & Dictoocha fibula Ehrenherg & & & * & & & & & \\
\hline
\end{tabular}




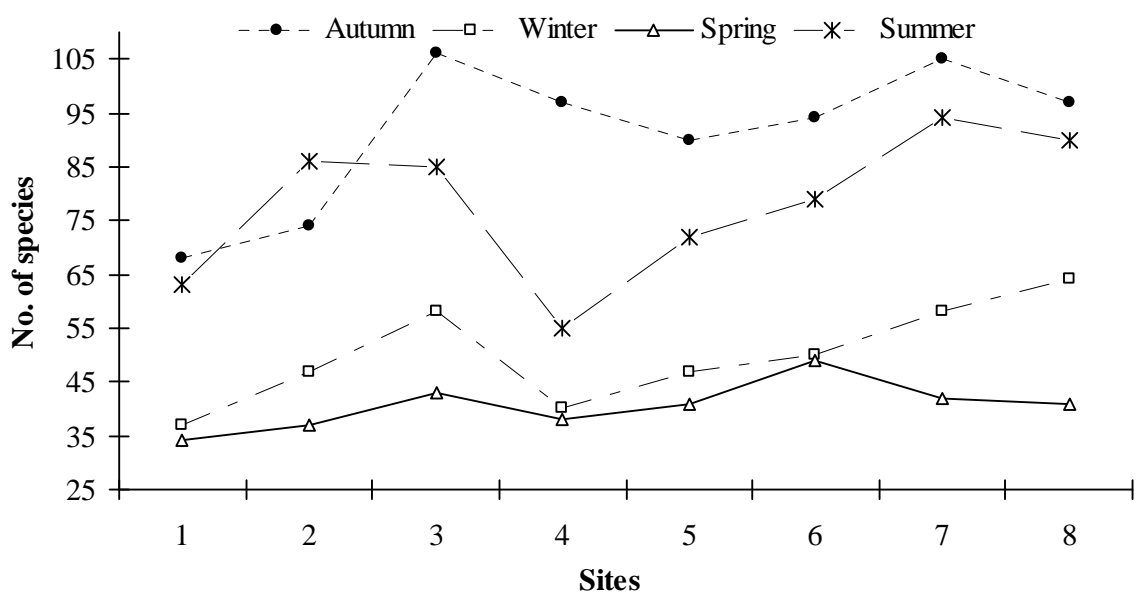

Fig. 2: The total number of phytoplankton species recorded at each site during autumn 2007-summer 2008.

The diversity and occurrence of the other groups (i.e. blue-green algae, coccolithophores and silicoflagellates) were very low. The standing crop of phytoplankton community was characterized by low density throughout the area of study, with an annual average of $4.5 \times 10^{3}$ individual/l. The spatial average of phytoplankton groups recorded throughout the whole study area during each season is shown in Fig. (3). On a seasonal scale, summer exhibited the highest phytoplankton cell count (average: $12.3 \times 10^{3}$ individual/l), while other seasons demonstrated low and close values with a minimum average value of $1.2 \times 10^{3}$ individual/l in autumn.

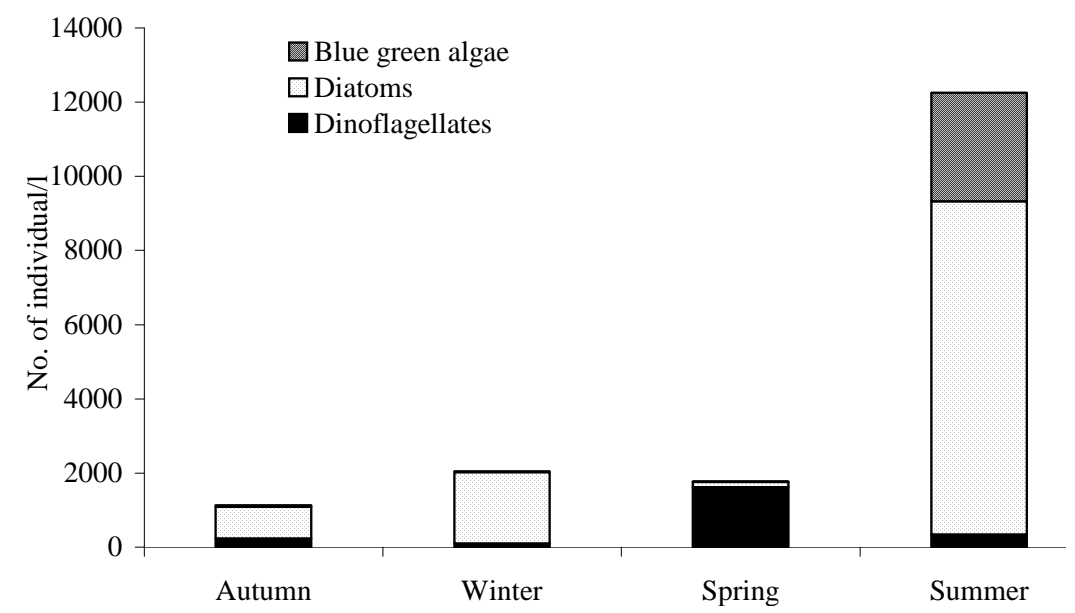

Fig. 3: Spatial average of phytoplankton groups (individual/l) throughout the whole study area during autumn 2007-summer 2008. 
The seasonal average standing crop of total phytoplankton at each site ranged between a minimum of $1.4 \times 10^{3}$ and a maximum of $7.7 \times 10^{3}$ individual /l at sites 8 and 4, respectively (Fig. 4).

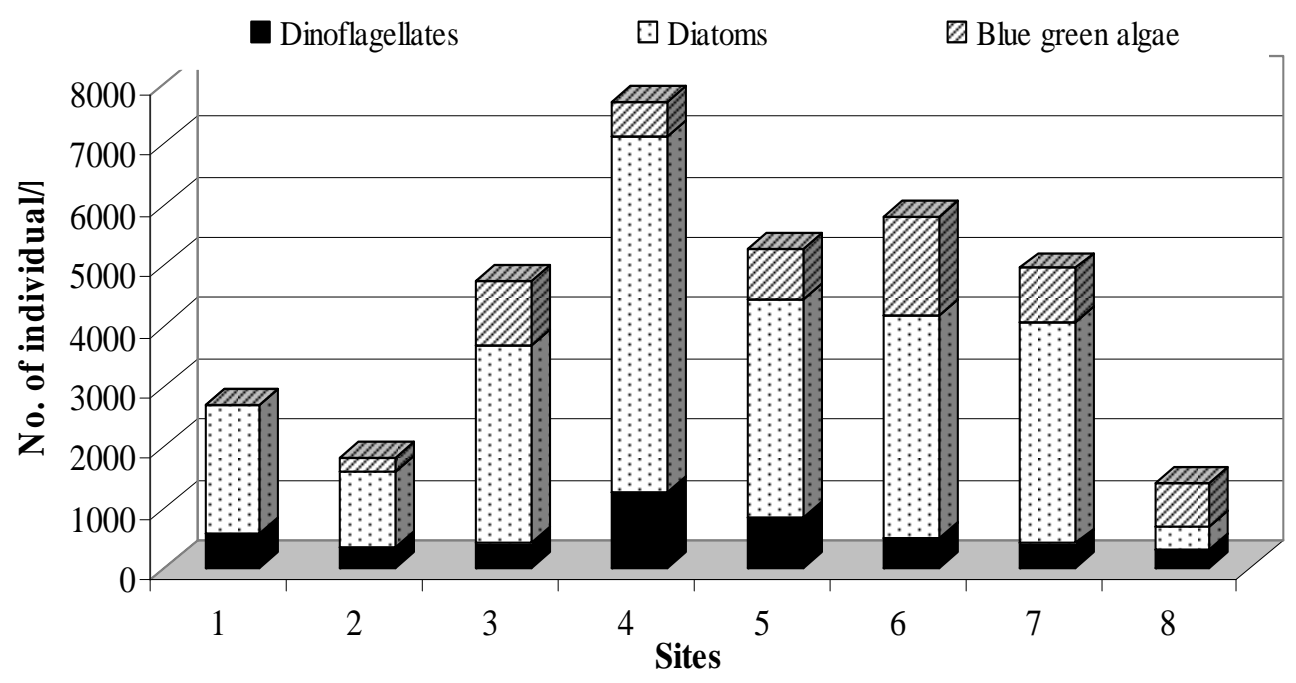

Fig. 4: Average seasonal density of phytoplankton groups (individual/l) at each sampling site during autumn 2007-summer 2008.

Diatoms showed approximately high densities at all sites comprising about 63.4-78.1\% of the total phytoplankton, except at site 8 (22.1\%). On the other hand, dinoflagellates predominated when the density of diatoms decreased. As shown in Fig. (5), the spatial distribution of phytoplankton standing crop demonstrated distinctive variations between the Gulf of Suez and both the Gulf of Aqaba and the southern Sinai Peninsula with different patterns of seasonal distribution. All sites which represent the southern part of Sinai Peninsula (3-6) showed relatively higher phytoplankton densities than that of both gulfs. A distinct peak of phytoplankton standing crop was observed in the southern part of Sinai Peninsula and the Gulf of Aqaba during summer with a maximum value of $23 \times 10^{3}$ individual $/ \mathrm{l}$ at site 4 but with lesser density in the Gulf of Aqaba $\left(4 \times 10^{3}\right.$ individual $\left./ \mathrm{l}\right)$. Conversely, the lowest abundance in the same area occurred during winter. In the Gulf of Suez, a relatively high phytoplankton production appeared during winter with a maximum value of $7 \times 10^{3}$ individual /l at site 1. 


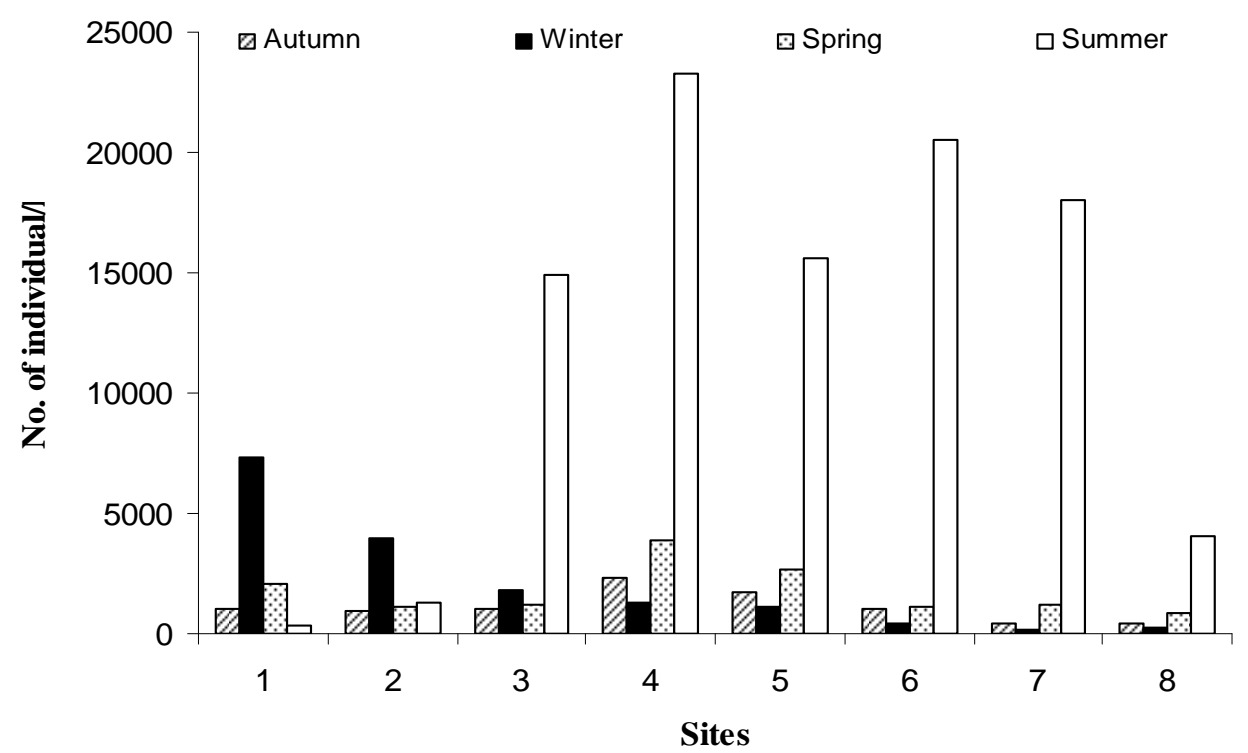

Fig. 5: Seasonal distribution of total phytoplankton standing crop (individual/l) at each sampling site.

The previously mentioned peak in summer is caused mainly by Hemiaulus hauckii and the two species of Trichodesmium. The first species represented about $73 \%$ of the total phytoplankton with an average of $9 \times 10^{3}$ individual/l. This species appeared with higher densities at sites 3-7. Trichodesmium constituted about $23.8 \%$ of the total count (average: $3 \times 10^{3}$ individual/l) with a maximum of $7 \times 10^{3}$ individual/l at site 6 . In autumn, relatively high production of different species appeared at different sites. For example, at sites 3-6, Nitzschia closterium and Pseudo-nitzschia delicatissima predominated the phytoplankton community comprising about $33.7 \%$ and $26.7 \%$ of the phytoplankton count, respectively. On the other hand, Rhizosolenia alata and $R$. calcar-avis showed relatively high count at sites 1 and 2 constituting about $22.3 \%$ and $16 \%$ of the total count, respectively. In winter, Rhizosolenia alata (44.6\%) and Pseudonitzschia delicatissima (19.2\%) continued with relatively high count at sites 1 and 2 accompanied with Thalassionema nitzschioides (30\%). Dinoflagellates showed the highest density in spring constituting $91.7 \%$ of the total count with an average of $2 \times 10^{3}$ individual/l. Prorocentrum and Ceratium were the most dominant genera. Of them Prorocentrum gracil, P. maximum, Ceratium contortum and C. candelabrum were the leader species with averages around $10^{3}$ individual $/ \mathrm{l}$.

The cluster analysis was performed based on seasonal average of phytoplankton standing crop recorded at each site (Fig. 5). It is observed that stations of the studied area combined to form distinct groups. The resulting 
dendrogram can be objectively partitioned into two groups at the level $\approx 60 \%$ of the similarity index. First group included sites 1 and 2, while another group is further divided into two clusters at the level $68.4 \%$ of the similarity index. The first cluster included sites 3-6 and the second formed from sites 7 and 8 . The dendrogram confirmed that the phytoplankton species composition and abundance in the Gulf of Suez differ greatly than that of the Gulf of Aqaba and the southern Sinai Peninsula. It is obvious that the similarity between the Gulf of Aqaba and the southern Sinai Peninsula was high (Fig. 6).

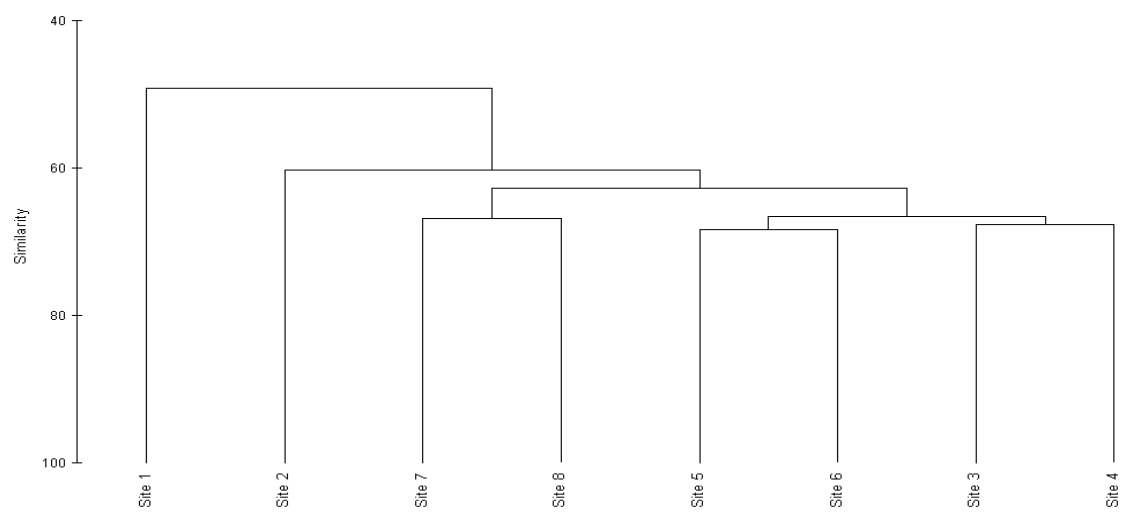

Fig. 6: Dendrogram, group average linkage, Bray Curtis of the similarities on abundance of different phytoplankton species between different sites.

\section{DISCUSSION}

In the area of study, physico-chemical conditions follow the normal seasonal fluctuations of the northern Red Sea. Water temperature values clearly reflected the subtropical conditions of the area as reported by Morcos (1970). Furthermore, the low concentrations of nutrient salts and chlorophyll $a$ indicated the oligotrophic condition of the investigated area as documented in the previous studies (Weikert, 1987). The high averages of nutrient salts and chlorophyll $a$ that recorded at the southern of Sinai Peninsula reflected the high values at sites 4 and 5 and this could be related to the using of this area as a mooring park for touristic and fishing boats.

Since the previous studies which described the phytoplankton population structure and distribution of the northern Red Sea is limited, the complete picture of phytoplankton species composition of this area has been generally underestimated. So, the present study comes to provide information on the spatial and seasonal distribution of phytoplankton in the neritic water of certain Egyptian Red Sea. During the present study, 181 species of phytoplankton were recorded (116 dinoflagellates, 61 diatoms). The recorded number of species in this study is considerably lower than that of the previous studies (283 species) as 
recorded by Skaikh et al., (1986). The relatively paucity of species number in the present work, particularly that of diatoms, in compared to dinoflagellates, may be related to the sampling strategy where samples were collected from the upper surface and/or from near shore waters. Moreover, the area of study is located north to $20^{\circ} \mathrm{N}$ which is characterized by lower diversity than the rest of the Red Sea as reported by Halim (1969) and Weikert (1987).

The recorded number of species at the southern of Sinai Peninsula (163 species, $90 \%$ of the total recorded species) was higher than that of the Gulf of Suez (133 species, 73\%) and the Gulf of Aqaba (141 species, 78\%). This is in agreement with the previous records which stated that the phytoplankton diversity of the Red Sea decrease gradually northward (Halim, 1969; Weikert, 1987). Most of the recorded species are cosmopolitan, tropical and sub-tropical forms, all of them are widely known in the Indo-Pacific region. Most of the dominant genera of phytoplankton assemblage (Ceratium, Protoperidinium, Chaetoceros, Rhizosolenia and Nitzschia) were more or less similar to other previous studies (Dowidar et al., 1978; Skaikh et al., 1986). Although the species number of dinoflagellates (116 species) exceeded that of diatoms (60 species), diatoms constituted the main bulk of phytoplankton community in terms of cell number. It contributed about $79.7 \%$ of the total phytoplankton with an annual average of $5.2 \times 10^{3}$ individual/l. Blue green algae and dinoflagellates occupied the second order in terms of cell count with relatively close annual averages ( 7.5 and $5.8 \times 10^{3}$ individual/l, respectively) comprising about 11.4 and $8.8 \%$ of the total phytoplankton, respectively.

Phytoplankton populations in the investigated area showed paucity in the number of individuals of each species. The standing crop of phytoplankton was relatively low ranging between less than $10^{3}$ individual/l and $23 \times 10^{3}$ individual/l (average $7 \times 10^{3}$ individual/l). This is comparable to other works in the Red Sea (Skaikh et al., 1986; Ismail, 2005) and considered as a general feature of tropical waters by some authors (Odum, 1971; Dowidar et al., 1978). In general, sites 4 and 5 showed higher abundance than others and this is probably related to the shallowness and sheltering condition of the bay in addition to its high nutrient concentrations resulting from different human activities at these sites. Our results revealed that summer was the most productive season in contrary with the previous studies which stated that late winter-early spring or the period from December-February were characterized by high phytoplankton production throughout (Halim, 1969; Levanon-Spanier et al., 1979; Skaikh et al., 1986). This could be explained by two reasons: the first is that the northern Red Sea (region north of $20^{\circ} \mathrm{N}$ latitude) is not notably influenced by the Arabian Ocean monsoon which affects the southern Red Sea (Edwards, 1987). The second is due to the neritic condition of the area under investigation.

In the present study, the high production during summer resulted from massive outbreak of the diatom Hemiaulus hauckii and the cyanobacterium genus Trichodesmium. The high production of Hemiaulus hauckii was 
previously recorded in The Gulf of Aqaba (Post et al., 2002), but is the first time to be reported in the southern of Sinai Peninsula region and this may be related to differ of time scale. The dominance of Hemiaulus hauckii and Trichodesmium during summer is usual in the oceanic water of tropical and subtropical regions (Gordon et al., 1994). On the other hand, it is obvious from the spatial distribution of phytoplankton that Gulf of Suez differs in the dominant species and timing of abundance from both Gulf of Aqaba and the southern of Sinai Peninsula. In the Gulf of Suez, Rhizosolenia alata and R. calcar-avis appeared with high densities during winter and this may be related to the difference in habitat and environmental conditions among the Gulf of Suez and both Gulf of Aqaba and the southern of Sinai Peninsula.

\section{REFERENCES}

Al-Najjar, D.; Badran, MI.; Richter, C.; Meyerhoefer, M. and Sommer, U. (2007). "Seasonal dynamics of phytoplankton in the Gulf of Aqaba, Red Sea”. Hydrobiologia, 579: 69-83.

Deyab, M. A.; Khedr, A.H.A. and El-Naggar, M.A. (2004). "Phytoplankton distribution in relation to environmental factors along the Suez Canal and the Red Sea coast of Egypt”. Algological Studies, 112: 123-140.

Dodge, J.D. (1982). "Marine Dinoflagellates of the British Isles". Her Majesty's Stationary Office, London, 303 pp.

Dowidar, N.; Raheem El-Din, S. and Aleem, A. (1978). "Pytoplankton populations in the region of Obhor, Jeddah, Saudi Arabia". Bull. Fac. Sci. King Abdull Aziz Univ., Jeddah, 2: 271-292.

Edwards, A.J. (1987). "Climate and oceanography". In: Edwards, A.J., Head, S.M. (Eds.), Key Environments: Red Sea. Pergamon Press, Oxford, pp. 45-70.

Field, J.G.; Clarke, K.R.and Warwick, R.M. (1982). "A practical strategy for analyzing multispecies distribution patterns". Mar. Ecol. Prog. Ser., 8: 3752.

Gordon, N.; Angel, DL.; Neori, A.; kres, N. and Kimor, B. (1994). "Heterotrophic dinoflagellates with symbiotic cyanobacteria and nitrogen limitation in the Gulf of Aqaba". Mar. Ecol. Prog. Ser., 107: 83-88.

Halim, Y. (1969)."Plankton of the Red Sea" Oceanography and Marine Biology, An Annual Review, 7: 231-275. 
Hendey, N.I. (1964). "An Introductory Account of the Smaller Algae of British Coastal Waters. Part V: Bacillariophyceae (Diatoms)". Her Majesty's Stationery Office, London, 317 pp.

Ismael, A.A. (2005). "Phytoplankton of the Gulf of Suez and the effect of ship traffic". J. Egypt. Acad. Soc. Environ. Develop., (D- Environmental studies), 6 (1): 75-92.

Kimor, B. and Golandsky, B. (1977). "Microplankton of the Gulf of Elat: aspects of seasonal and bathymetric distribution". Mar. Biol., 42: 55-67.

Levanon-Spanier, J.; Padan, E. and Reiss, Z. (1979). "Primary production in a desert-enclosed sea - the Gulf of Aqaba, Red Sea". Deep-Sea Res., 26: 673-685.

Morcos, S. A. (1970). "Physical and chemical oceanography of the Red Sea". Oceanography Marine Biology Annual Review, 8: 73-202.

Odum, E.P. (1971). "Fundamentals of ecology". Saunders Comp., London, 459pp.

Parsons, T.R.; Maita, Y. and Lalli, C.M. (1984). "A Manual of Chemical and Biological Methods for Seawater Analysis". Pergamon Press, Oxford, 173 pp.

Pätzold, J.; Halbach, P. E.; Hempel, G. and Weikert; H. (2000). "MeteorBerichte: Ostliches Mittelmeer-Nordliches Rotes Meer 1999 Cruise No. 44. 00-3". Leitstelle METEOR: Institut fur Meereskunde der Universitat Hamburg, Hamburg.

Post, A.F.; Dedej, Z.; Gottlieb, R.; Li, H.; Thomas, DN.; El-Absawi, M.; El-Naggar, A.; El-Gharabawi, M. and Sommer, U. (2002). "Spatial and temporal distribution of Trichodesmium spp. in the stratified Gulf of Aqaba, Red Sea". Mar. Ecol. Prog. Ser., 239: 241-250.

Shaikh, E.A.; Roff, J. C. and Dowidar, N. M. (1986). "Phytoplankton ecology and production in the Red Sea off Jiddah, Saudi Arabia". Mari Biol., 92: 405- 416.

Sommer, U. (2000). "Scarcity of medium-sized phytoplankton in the Red Sea explained by strong bottom-up and weak top-down control". Mar. Ecol. Prog. Ser., 197:19-25. 
Sommer, U.; Berninger, U.G.; Böttger-Schnack, R.; Cornils, A.; Hagen, W.; Hansen, T.; Al-Najjar, T.; Post, A.F.; Schnack-Schiel, S.B.; Stibor, H.; Stübing, D. and Wickham, S. (2002). "Grazing during early spring in the Gulf of Aqaba and the northern Red Sea". Mar. Ecol. Prog. Ser., 239:251261.

Sournia, A. (1977). "Notes on primary productivity of coastal waters in the Gulf of Eilat (Red Sea)". Int. Rev. Gesamt. Hydrobiol., 62: 813-819.

Taylor, F.J.R. (1976). "Dinoflagellates from the international Indian Ocean Expedition”. Bibl. Botanica, 132: 1-234.

Tomas, C.R. (1996). "Identifying Marine Diatoms and Dinoflagellates". Academic Press, California.

Touliabah, H. E.; Abu El-Kheir, W.S.; Kuchari, M. G. and Abdulwassi, N.I.H. (2010). "Phytoplankton composition at Jeddah coast-Red Sea, Saudi Arabia in relation to some ecological factors”. JKAU: Sci., 22 (1):115131.

Utermöhl, H. (1958). "Zur Vervollkommung der quantitativen Phytoplankton methodic". Mitt. Int. Verein. Limnol.: pp.1-38.

Weikert, H. (1987). "Plankton and the pelagic environment". In: Edwards, A.J., Head, S.M. (Eds.), Key Environments: Red Sea. Pergamon Press, Oxford, pp. 90-111. 